\title{
Correction to: Teaching in Different Spaces - How Professionals Work Together in All-Day Schools in Switzerland and in Leisure Time Centres in Sweden
}

Patricia Schuler Braunschweig, Helene Elvstrand, Christa Kappler, Lina Lago, Magnus Jansson and Emanuela Chiapparini

\section{Correction to: \\ Chapter 7 in: M. Schüpbach and N. Lilla (eds.), Extended Education from an International Comparative Point of View, https://doi.org/10.1007/978-3-658-27172-5_7}

In the original version of this chapter the author's name was inadvertently entered in chapter xml as first name: Patricia Schuler and surname: Braunschweig, this has now been corrected as first name: Patricia and surname: Schuler Braunschweig. 